\title{
A Novel Analytical Framework for Dynamic Quaternion Ship Domains
}

\author{
Ning Wang \\ (Marine Engineering College, Dalian Maritime University, China) \\ (E-mail: n.wang.dmu.cn@gmail.com)
}

In this paper, a novel analytical framework for Dynamic Quaternion Ship Domain (DQSD) models has been initially proposed via the Quaternion Ship Domain (QSD) model structure. Unlike previous ship domains, the proposed DQSD model is able to capture essential subjectivity and objectivity of ship domains. To be specific, the significant characteristics are as follows:

(1) The proposed DQSD model is integrated by three independent submodels of ship, human and circumstance, which are determined by ship manoeuvrability, navigator's states, and navigation circumstance, respectively.

(2) The ship manoeuvrability derived from the MMG-type ship motion model is employed to establish the ship submodel which identifies the DQSD scale.

(3) A novel navigator reliability model is proposed to realize the human submodel which defines the ship domain shape with navigator ability, physical and mental states being input variables.

(4) In addition, visibility, wind force, wave and traffic congestion are incorporated into the circumstance submodel which is employed to zoom in or out of the DQSD-type ship domain.

Finally, the well-known Esso Osaka tanker model is used to conduct simulation studies on various typical stationary and dynamic situations, and comparative investigations with each other have been comprehensively analysed. Simulation results demonstrate that the DQSD model can capture critical dynamics of ship domains and undoubtedly be effective and superior to previous ship domains in terms of performance and accuracy.

\section{KEY WORDS}
1. Dynamic Quaternion Ship Domain.
2. Ship Manoeuvrability.
3. Human Factor
4. Circumstance.

Submitted: 9 April 2012. Accepted: 6 September 2012. First published online: 25 October 2012.

1. INTRODUCTION. It is well known that ship domains are powerful and fundamental approaches to dealing with ship navigation risk assessment (Gucma and Pietrzykowski, 2006; Pietrzykowski, 2008; Szlapczynski, 2006, 2008; Wang, 2010; Qu et al., 2011), with collision avoidance (Hwang, 2002; Kao et al., 2007; Pedersen and 
Inoue, 2003; Wilson et al., 2003), with marine traffic simulations (Lisowski et al., 2000) and with optimal trajectory planning (Smierzchalski and Michalewicz, 2000), etc. In the past decades, many researchers (Fujii and Tanaka, 1971; Goodwin, 1975; Davis, 1980, 1982; Coldwell, 1983; Zhao et al., 1993; Zhu et al., 2001; Smierzchalski, 2001; Kijima and Furukawa, 2001, 2003; Pietrzykowski and Uriasz, 2004, 2006, 2009; Pietrzykowski, 2008) have presented various ship domains with different dimensions and shapes. Briefly, for clarity, the innovation of ship domains could be roughly divided into three methodologies, i.e., statistical, individual and intelligent models. The seminal results of statistical ship domain have been proposed by Fujii (Fujii and Tanaka, 1971) and Goodwin (Goodwin, 1975), whereby ellipse and circle ship domains are derived from statistical data, respectively. Davis (Davis, 1980) and Coldwell (Coldwell, 1983) further developed the Goodwin and Fujii models, by taking into account the COLREGS and boundary continuity respectively. Recently, Pietrzykowski (Pietrzykowski and Uriasz, 2009) proposed the polygon ship domain defined by statistical safety distances in different relative bearing of target ships. In this case, this model could be considered as the discrete version of Davis model. It should be noted that the statistical models focus on macro identification for common ship domains rather than individual characteristics of different ships under varying circumstances. Reasonably, it is followed by individual ship domains, which are dependent on specific ship parameters and dimensions. Smierzchalski (Smierzchalski and Michalewicz, 2000) proposed a hexagon ship domain, whereby the ship speed and manoeuvrability was roughly considered by a series of empirical formulae. However, the complex model without explicitly analytical and systematic identification methods would hinder its practical applications. In addition, Kijima (Kijima and Furukawa, 2001) presented 'block and watch' areas for ship domains by combining two semiellipses as fore and aft sectors, respectively. Note that the radii of block and watch areas are roughly calculated by taking into account turning circle dynamics. Alternatively, artificial neural networks are used by Lisowski (Lisowski et al., 2000) and Zhu (Zhu et al., 2001) to identify ship domain models from collected data considering predefined input variables, i.e., speed, bearing, course and distance, etc. Pietrzykowski (Pietrzykowski, 2008) provided the ship domain with fuzzy boundaries by using fuzzy systems to investigate the ship domain based risk assessment. Note that these intelligent ship domains could be archived as approximation of statistical models since the resultant 'black-box' models stem from statistical data. Unfortunately, significant factors, i.e., human and circumstance influences, have not yet been incorporated into abovementioned ship domains.

Essentially, these previous models are described in geometrical manner and reasonably classified into three types, i.e., circle, ellipse and polygon ship domains (Liu et al., 2011). It is obvious that there are no systematic and analytical principles for identification and design of these ship domains since abovementioned ship domains are actually subjective and qualitative models. The main reasons could be concluded as follows:

- Different factors considered while assessing navigational situations would determine different shapes and dimensions of ship domains.

- Most ship domains are estimated by statistical methods based on plenty of questionnaire data or simulator trials which would lead to complex shapes (Pietrzykowski and Uriasz, 2009). 
In order to circumvent the foregoing problems, a unified analytical framework for describing ship domain models has been presented by Wang (Wang et al., 2009). This framework provides a unified platform for previous ship domains, which could be considered as the generalized model of geometrical ship domains. However, these transformed analytical ship domains are still inflexible and lack a systematic design.

In addition, according to the general definition of ship domains "The surrounding effective waters which the navigator of a ship wants to keep clear of other ships or fixed objects" (Goodwin, 1975), the navigator and circumstance would play critical roles in determination of ship domain for individual ship (either own or target ship). As a consequence, how to estimate the required water area for a ship we considered becomes an involved task of model identification for individual ship domain. It is rationally highlighted that the concept of 'domain', in the series of our work, is interpreted as the fundamental model for individual ship domain dependent on the information about human, ship and circumstance. In other words, we consider the 'domain' as the model of the large-scale system integrated by navigator states, circumstance influences and manoeuvring dynamics of the ship considered, whereas other ships or fixed objects within encountering situations are excluded in this model. In this case, the ship domain we considered could be reasonably taken as the general model contributing to comprehensive research fields (i.e., risk assessment, traffic capacity and path planning, etc.) rather than the specific one restricted to a single task (i.e., collision avoidance).

As innovative contributions in our previous work (Wang, 2010), novel ship domain models termed Quaternion Ship Domain (QSD) and Fuzzy Quaternion Ship Domain (FQSD) have been proposed to establish a model structure for ship domain identification in an analytical and systematic manner. To be specific, the dimensions of ship domain are defined by fore, aft, starboard and port radii, respectively, and the shape is modelled by another index parameter $k$. Note that these parameters are only subject to ship manoeuvrability (Wang et al., 2011), whereas human factors and circumstance states have not been incorporated into this model framework. Therefore, an effective methodology would be desired to systematically and analytically identify QSD-type ship domain models which could well enclose abovementioned influence factors imposed on ship domains.

In this paper, we initially propose an analytical framework to implement a novel Dynamic Quaternion Ship Domain (DQSD) model which could sufficiently consider ship, human and circumstance factors based on the QSD-type model structure. To be specific, the proposed DQSD model is identified by three parts of parameters, i.e., scale vector, shape parameter and zooming coefficient, which are determined by ship manoeuvrability, navigator's states, and navigation circumstance, respectively. It follows that the human, ship and circumstance submodels of the DQSD are well defined by carefully considering pervasive influence factors, respectively. Furthermore, the entire DQSD model integrated by abovementioned submodels could be well implemented in an analytical framework for ship domains. As a consequence, the proposed analytical framework for DQSD model can dynamically identify objective and subjective information from the aspects of human, ship and circumstance and thereby result in an adequate candidate for complex ship domain identifications. Finally, simulation studies conducted on the well-known Esso Osaka tanker model in typical stationary and dynamic situations. The results demonstrate the effectiveness of the analytical framework for DQSD 
models which can achieve essential dynamics of ship domains. It is evident that the proposed methodology for DQSD models is able to provide the field of marine traffic engineering with theoretical and practical foundations.

2. QUATERNION SHIP DOMAIN (QSD). The definition of ship domains (Goodwin, 1975) implies that ship domains are qualitative and quantitative models incorporated with both objective and subjective characteristics. However, most previous ship domain models pursue qualitative investigations; in addition, the subjective ship domains that navigators preserve are usually distinct, with objective models that corresponding vessels should hold. Fortunately, Wang (Wang, 2010) proposed a novel Quaternion Ship Domain (QSD) which aims to develop a systematic and analytical approach to a unified framework for modelling ship domains. Unlike the previous circle, ellipsoid and polygon ship domains, the QSD model is characterized by the scale quaternion $Q=\left\{R_{\text {fore }}, R_{\text {aft }}, R_{\text {port }}, R_{\text {starb }}\right\}$ and the shape parameter $k$, whereby $Q$ indicates the radii of fore, aft, starboard and port directions of own ship. The formulae could be rewritten as follows:

$$
\begin{gathered}
Q S D_{k}=\left\{(x, y) \mid f_{k}(x, y ; Q) \leqslant 1, Q=\left\{R_{\text {fore }}, R_{\text {aft }}, R_{\text {starb }}, R_{\text {port }}\right\}, k \geqslant 1\right\} \\
f_{k}(x, y ; Q)=\left(\frac{2 x}{(1+\operatorname{sgn}(x)) R_{\text {fore }}-(1-\operatorname{sgn}(x)) R_{\text {aft }}}\right)^{k} \\
+\left(\frac{2 y}{(1+\operatorname{sgn}(y)) R_{\text {starb }}-(1-\operatorname{sgn}(y)) R_{\text {port }}}\right)^{k}
\end{gathered}
$$

where $\operatorname{sgn}($.$) is a sign function defined in Equation (3):$

$$
\operatorname{sgn} x=\left\{\begin{array}{cc}
1, & x \geqslant 0 \\
-1, & x<0
\end{array}\right.
$$

In addition, Wang (Wang, 2010) determined the $Q$ and $k$ parameters, by considering parts of the dynamic and stationary affecting factors via following nonlinearities:

$$
\left\{\begin{array}{l}
R_{\text {fore }}=\left(1+1.34 \sqrt{k_{A D}^{2}+\left(k_{D T} / 2\right)^{2}}\right) L \\
R_{\text {aft }}=\left(1+0.67 \sqrt{k_{A D}^{2}+\left(k_{D T} / 2\right)^{2}}\right) L \\
R_{\text {starb }}=\left(0.2+k_{D T}\right) L \\
R_{\text {port }}=\left(0.2+0.75 k_{D T}\right) L
\end{array}\right.
$$

where:

$L$ is the own ship length.

$k_{A D}$ and $k_{D T}$ represent gains of the advance $A_{D}$ and the tactical diameter $D_{T}$ respectively, and can be calculated as follows:

$$
\left\{\begin{array}{l}
k_{A D}=A_{D} / L=10^{0.3591 \lg V_{\text {own }}+0.0952} \\
k_{D T}=D_{T} / L=10^{0.5441 \lg V_{\text {own }}-0.0795}
\end{array}\right.
$$

where $V_{\text {own }}$ is the own ship speed represented in knots. 
3. DYNAMIC QUATERNION SHIP DOMAIN (DQSD). Actually, the scale and shape parameters of the QSD model could be considered as quantitative and qualitative identifications, respectively. Varieties of affecting factors could be efficiently incorporated into this model framework to determine quantitative and qualitative characteristics simultaneously. As a consequence, the subjective and objective features could be merged into a unified model. However, so far, effective identification methods for scale and shape parameters have not been presented to sufficiently build the nonlinear relationship between inputs (i.e., including human, ship and environment factors), and outputs (i.e., ship domain parameters). To be specific, ship domain parameters $Q$ and $k$ should be carefully characterized by human factors, ship manoeuvrability and traffic circumstance, respectively. In order to implement the complete QSD model by considering the abovementioned factors, the whole ship domain termed Dynamic Quaternion Ship Domain (DQSD) could be described as follows:

$$
\operatorname{DQSD}(t)=f(C(\mathbf{\Omega}(t)) \mathbf{Q}(\boldsymbol{\Theta}(t)), k(\boldsymbol{\Xi}(t)))
$$

where:

The nonlinear function $f($.$) defines the DQSD boundary given by Equation (2).$

$C($.$) is the scalability function evaluating the traffic circumstance.$

$\mathbf{Q}($.$) is quaternion scale vector function, i.e. \mathbf{Q}(\boldsymbol{\Theta}(t))=\left[R_{\text {fore }}(\boldsymbol{\Theta}(t)), R_{a f t}(\boldsymbol{\Theta}(t))\right.$, $\left.R_{\text {port }}(\Theta(t)), R_{\text {starb }}(\Theta(t))\right]^{\mathrm{T}}$, of ship manoeuvrability.

$C(.) \mathbf{Q}($.) denotes the scalable quaternion vector.

$k($.$) is the shape function dependent on ship navigator's states.$

$\boldsymbol{\Omega}(t), \boldsymbol{\Theta}(t)$ and $\boldsymbol{\Xi}(t)$ are time-varying environment, ship manoeuvrability and human state vectors including corresponding variables, respectively.

3.1. The Ship Submodel. In order to identify ship factors affecting the DQSD model, we first establish the ship manoeuvrability prediction model by incorporating ship motion models. To be specific, the MMG ship motion model is used to build the second order response manoeuvre model for ship manoeuvrability predictions since the $K$ and $T$ parameters in a Nomoto-type model are directly related to the ability of course changing and keeping. In this case, the ship submodel of DQSD model would be reasonably generated on the basis of ship manoeuvrability.

The MMG ship motion model could be described as follows:

$$
\left\{\begin{array}{l}
\left(m+m_{x}\right) \dot{u}-\left(m+m_{y}\right) v r=X_{H}+X_{P}+X_{R} \\
\left(m+m_{y}\right) \dot{v}+\left(m+m_{x}\right) u r=Y_{H}+Y_{P}+Y_{R} \\
\left(I_{z z}+J_{z z}\right) \dot{r}=N_{H}+N_{P}+N_{R}
\end{array}\right.
$$

where:

$m$ and $I_{z z}$ are mass and moment of inertia of the ship, respectively.

$m_{x}, m_{y}$ and $J_{z z}$ denote added mass in the $x$ - and $y$-directions and added moment of inertia about $z$-axis, respectively.

$X, Y$ and $N$ denote surge forces, sway forces and yaw moments, respectively, due to hydrodynamics, propeller and rudder denoted by subscripts $H, P$ and $R$.

$u$ and $v$ are the velocities along $X$ (towards forward) and $Y$ axes (towards starboard) respectively.

$r$ is the yaw rate. 
Apply linearization operation to Equation (7) and omit the high order terms, this becomes:

$$
\left\{\begin{array}{l}
\left(m+m_{x}\right) \Delta \dot{u}-\left(m+m_{y}\right) v r=X_{u} \Delta u \\
\left(m+m_{y}\right) \dot{v}+\left(m+m_{x}\right) u_{0} r=Y_{v} v+Y_{r} r+Y_{\delta} \delta \\
\left(I_{z z}+J_{z z}\right) \dot{r}=N_{v} v+N_{r} r+N_{\delta} \delta
\end{array}\right.
$$

Without loss of generality, assume that the forward speed $u$ keeps constant. Applying Laplace transformation and inverse operation, we obtain the second order response model as follows:

$$
\left\{\begin{array}{l}
T_{1} T_{2} \ddot{r}+\left(T_{1}+T_{2}\right) \dot{r}+r=K\left(\delta+T_{3} \dot{\delta}\right) \\
T_{1} T_{2} \ddot{v}+\left(T_{1}+T_{2}\right) \dot{v}+v=K_{v}\left(\delta+T_{3 v} \dot{\delta}\right)
\end{array}\right.
$$

where the corresponding non-dimensional hydrodynamics derivatives could be determined by the following equations:

$$
\left\{\begin{array}{l}
T_{1} T_{2}=\left(\frac{L}{V}\right)^{2} \frac{\left(m^{\prime}+m_{y}^{\prime}\right)\left(I_{z z}^{\prime}+J_{z z}^{\prime}\right)}{C^{\prime}} \\
T_{1}+T_{2}=\left(\frac{L}{V}\right) \frac{-\left(m^{\prime}+m_{y}^{\prime}\right) N_{r}^{\prime}-\left(I_{z z}^{\prime}+J_{z z}^{\prime}\right) Y_{v}^{\prime}}{C^{\prime}} \\
K=\left(\frac{V}{L}\right) \frac{N_{v}^{\prime} Y_{\delta}^{\prime}-N_{\delta}^{\prime} Y_{v}^{\prime}}{C^{\prime}} \\
T_{3}=\left(\frac{L}{V}\right) \frac{\left(m^{\prime}+m_{y}^{\prime}\right) N_{\delta}^{\prime}}{N_{v}^{\prime} Y_{\delta}^{\prime}-N_{\delta}^{\prime} Y_{v}^{\prime}} \\
K_{v}=\left(\frac{V}{L}\right) \frac{N_{r}^{\prime} Y_{\delta}^{\prime}-\left(Y_{r}^{\prime}-\left(m^{\prime}+m_{x}^{\prime}\right)\right)}{C^{\prime}} \\
T_{3 v}=\left(\frac{L}{V}\right) \frac{Y_{\delta}^{\prime}\left(I_{z z}^{\prime}+J_{z z}^{\prime}\right)}{N_{r}^{\prime} Y_{\delta}^{\prime}-\left(Y_{r}^{\prime}-\left(m^{\prime}+m_{x}^{\prime}\right)\right)} \\
C^{\prime}=N_{r}^{\prime} Y_{v}^{\prime}-N_{v}^{\prime}\left(Y_{r}^{\prime}-\left(m^{\prime}+m_{x}^{\prime}\right)\right)
\end{array}\right.
$$

where:

$L$ denotes ship length.

$$
V=\left(u^{2}+v^{2}\right)^{0 \cdot 5} \text { is ship speed. }
$$

Other coefficients are non-dimensional hydrodynamics derivatives which can be derived from ship dynamic and stationary parameters $\boldsymbol{\Theta}(t)=\left[\boldsymbol{\Theta}_{S t a}, \boldsymbol{\Theta}_{D y n}(t)\right]^{\mathrm{T}}$, where stationary parameter vector $\boldsymbol{\Theta}_{S t a}$ mainly includes ship length $L$, width $B$, block coefficient $C_{b}$, draft d, displacement $\nabla$, rudder area $A_{R}$, height of rudder $H_{R}$ and propeller diameter $D_{P}$, etc., while dynamic parameter vector $\boldsymbol{\Theta}_{D y n}(t)$ dependent on time mainly consists of surge speed $u$, sway speed $v$, yaw angle speed $r$, shaft revolution $n$ and rudder angle $\delta$, etc.

Ship manoeuvrability is usually determined by course changing and keeping ability which is critically characterized by the turning diameter $D$ and advance at 90 degree heading $A_{d}$ obtained by:

$$
\frac{D(\Theta(t))}{L}=\left(\frac{V(t)}{L}\right) \frac{2}{\left|K(\Theta(t)) \delta_{0}(t)\right|}, \delta_{0}(t) \neq 0
$$




$$
\begin{aligned}
\frac{A_{d}(\Theta(t))}{L}= & \left(\frac{V(t)}{L}\right) \\
& \times\left(T_{1}(\boldsymbol{\Theta}(t))+T_{2}(\boldsymbol{\Theta}(t))-T_{3}(\boldsymbol{\Theta}(t))+\frac{t_{1}(t)}{2}+\frac{1}{\left|K(\Theta(t)) \delta_{0}(t)\right|}\right), \delta_{0}(t) \neq 0
\end{aligned}
$$

where:

$\delta_{0}(t)$ denotes command rudder angle in the process of turning circle manoeuvre.

$t_{1}(t)$ is the time for the rudder turning from amidships (zero) to the command angle $\delta_{0}(t)$, and can be derived from the model of steering characteristics as follows:

$$
T_{E} \dot{\delta}+\delta=\delta_{0}(t), \text { where }|\delta|,\left|\delta_{0}\right| \leqslant 35^{\circ} \text { and }|\dot{\delta}| \leqslant 3^{\circ} / s
$$

where $T_{E}$ is steering time constant and usually chosen as $2 \cdot 5$ seconds.

Rationally, we can select the ship dynamic domain boundary as the maximum area covered by the ship with $90^{\circ}$ course changing with time-varying speed $V(t)$ and command rudder angle $\delta_{0}(t)$. However, note that $D(\Theta(t))$ and $A_{d}(\Theta(t))$ would become infinity if $\left|\delta_{0}(t)\right|$ tends to zero. It implies that the obtained $D(\Theta(t))$ and $A_{d}(\boldsymbol{\Theta}(t))$ would be meaningless when $\left|\delta_{0}(t)\right|$ keeps less than a threshold $\Delta$ (i.e. $5^{\circ}$ ). In order to circumvent this problem, we restrict the DQSD boundary partially dependent on $\left|\delta_{0}(t)\right|$ to be the one determined by the threshold $\Delta$ whenever the command rudder angle $\left|\delta_{0}(t)\right|$ is less than $\Delta$. As a consequence, the ship submodel $\mathbf{Q}(\boldsymbol{\Theta}(t))$ could be described as follows:

$$
\boldsymbol{Q}(\boldsymbol{\Theta}(t))=\left[R_{\text {fore }}(\boldsymbol{\Theta}(t)), R_{\text {aft }}(\boldsymbol{\Theta}(t)), R_{\text {starb }}(\boldsymbol{\Theta}(t)), R_{\text {port }}(\boldsymbol{\Theta}(t))\right]^{\mathrm{T}}
$$

where:

$$
\left\{\begin{array}{l}
R_{\text {fore }}(\boldsymbol{\Theta}(t))=L+A_{d}(\boldsymbol{\Theta}(t)) \\
R_{\text {aft }}(\boldsymbol{\Theta}(t))=L+\frac{1}{2} D(\boldsymbol{\Theta}(t)) \\
R_{\text {starb }}(\boldsymbol{\Theta}(t))=B+\frac{1}{2} D(\boldsymbol{\Theta}(t)) \\
R_{\text {port }}(\boldsymbol{\Theta}(t))=B+\frac{1}{2} \alpha D(\boldsymbol{\Theta}(t))
\end{array}\right.
$$

where:

$0<\alpha \leqslant 1$ is the predefined coefficient which is used to sufficiently consider the influence from the International Regulations for Preventing Collisions at Sea (COLREGS) rules.

Time-varying parameters $D(\Theta(t))$ and $A_{d}(\Theta(t))$ could be determined by Equations (11) and (12), respectively.

3.2. The Human Factor Submodel. Essentially, ship domain models possess subjectivity which would be subject to human factors (i.e., navigator's influences) to some extent. In this case, human reliability analysis is the reasonable candidate to be considered to contribute to the human factor submodel of DQSD model. The popular 
human reliability model dependent on time can be described as follows:

$$
R(t)=\exp \left(-\int_{0}^{t} E(\tau) d \tau\right)
$$

where, $E(t)$ denotes behaviour error rate which can be a constant or time dependent function.

Usually, $E(t)$ is selected as a Weibull probability density function given by:

$$
E(t)=\frac{\beta}{\eta}\left(\frac{t}{\eta}\right)^{\beta-1} \exp \left(-(t / \eta)^{\beta}\right)
$$

where, $\beta$ and $\eta$ are shape and scale parameters of the Weibull function, respectively. Substituting Equation (17) into Equation (16), we obtain:

$$
R(t)=\exp \left(\exp \left(-(t / \eta)^{\beta}\right)-1\right)
$$

However, the above mentioned function at Equation (18) is time dependent rather than human states dependent. Note that the time variable in Equation (18) could be substituted by a virtual scale variable $\rho$, which is determined by the navigator's state variables. As a consequence, the navigator reliability model could be established as follows:

$$
\begin{gathered}
R(\boldsymbol{\Xi}(t))=\exp \left(\exp \left(-\left(\frac{\rho(\boldsymbol{\Xi}(t))}{\eta}\right)^{\beta}\right)-1\right) \\
\rho=\boldsymbol{\Gamma}^{\mathrm{T}} \boldsymbol{\Xi}(t)
\end{gathered}
$$

where:

$\Xi(t)=\left[\xi_{1}(t), \xi_{2}(t), \ldots, \xi_{n}(t)\right]^{\mathrm{T}} \in R^{n}\left(-1 \leqslant \xi_{i}(t) \leqslant 0\right)$ is the ship navigator state vector including skill ability, physical and mental states, etc., which would be timevarying variables.

$\boldsymbol{\Gamma}=\left[\gamma_{1}, \gamma_{2}, \ldots, \gamma_{n}\right]^{\mathrm{T}} \in R^{n}$ is the weight vector with compatible dimensionality satisfying that $0<\gamma_{i}<1$.

Based on the navigator reliability model, the human factor submodel of DQSD could be implemented by:

$$
k(\boldsymbol{\Xi}(t))=\frac{1}{\exp \left(\exp \left(-\left(\boldsymbol{\Gamma}^{\mathrm{T}} \boldsymbol{\Xi}(t) / \eta\right)^{\beta}\right)-1\right)}
$$

where the scale and shape parameters could be reasonably selected as $\eta=\|\boldsymbol{\Gamma}\|_{1}$ and $\beta=1$, respectively.

3.3. The Circumstance Submodel. In order to consider weather conditions and navigation circumstances which would rationally contribute to the proposed DQSD model, we consider the circumstance submodel to complete the whole DQSD model. Intuitively, we describe the environment states as vector $\boldsymbol{\Omega}(t)=\left[\omega_{1}(t), \omega_{2}(t), \ldots\right.$, $\left.\omega_{n}(t)\right]^{\mathrm{T}}$, where $\omega_{i}(t)$ denotes time-varying environment states including visibility, wind force, wave and traffic congestion, etc. Note that these dynamic environmental state variables are essentially qualitative and imprecise knowledge obtained from artificial assessment, which cannot be well identified by traditional methods. To the contrary, 
fuzzy systems are usually considered as effective tools for dealing with uncertainty information. In this case, the circumstance submodel with navigation environment assessment being output could be well modelled by using fuzzy sets theory. To be specific, all the input variables $\omega_{i}(t)$ are normalized into the unity interval $[0,1]$ and labelled by corresponding fuzzy sets $A_{i j}\left(\omega_{i}(t)\right)$ described Gaussian membership functions:

$$
A_{i j}\left(\omega_{i}(t)\right)=\exp \left(\frac{\left(\omega_{i}(t)-c_{i j}\right)^{2}}{\sigma_{i j}^{2}}\right)
$$

where, $\omega_{i}(t), i=1,2,3,4$, denote environment states including visibility, wind force, wave and traffic congestion, respectively.

The output variable, environment assessment, denoted by $C(\mathbf{\Omega}(t))$ falls into the interval $[1-\zeta, 1+\zeta]$ around one, whereby $0<\zeta<1$. For simplicity, we consider each input variable is uniformly partitioned by only two fuzzy sets, respectively, i.e. $c_{i j} \in\{0$, 1 ) and $\sigma_{i j}=0 \cdot 5, j=1,2$. And, the output variable is uniformly partitioned by five fuzzy sets $B_{k}, k=1,2, \ldots, 5$, which are singletons, i.e. $B_{k} \in\{0 \cdot 6,0 \cdot 8,1,1 \cdot 2,1 \cdot 4\}$. Hence, fuzzy rules could be intuitively described as follows:

Rule 1: If $\omega_{1}(t)$ is $A_{1 j_{1}}\left(\omega_{1}(t)\right)$ and ... and $\omega_{4}(t)$ is $A_{4 j_{4}}\left(\omega_{1}(t)\right)$, Then $C(\boldsymbol{\Omega}(t))$ is $B_{k_{l}}$

It follows that the whole fuzzy systems for modelling the circumstance submodel could be established as follows:

$$
C(\boldsymbol{\Omega}(t))=\frac{\sum_{l} B_{k_{l}} \varphi_{l}(\boldsymbol{\Omega}(t))}{\sum_{l} \varphi_{l}(\mathbf{\Omega}(t))}
$$

where $\varphi_{l}$ is the aggravated activation function given by:

$$
\phi_{l}(\mathbf{\Omega}(t))=\prod_{i=1}^{4} A_{i, j_{i}}\left(\omega_{i}(t)\right)
$$

3.4. Remark 1. It should be noted that the whole DQSD model at Equation (6) is defined by three time-dependent functions, i.e., shape index $k(t)$, scale quaternion vector $\mathbf{Q}(t)$ and scalability factor $C(t)$, which are determined by human, ship and circumstance submodels given by Equations (14), (15), (21) and (24), respectively. In this case, we consider the DQSD as the model of the large-scale system integrated by navigator states, circumstance influences and manoeuvring dynamics of the ship considered, whereas other ships or fixed objects within encountering situations are excluded in this model. Reasonably, the DQSD we proposed could be taken as the general model contributing to comprehensive research fields (i.e., risk assessment, traffic capacity and path planning, etc.), rather than the specific one restricted to single task (i.e., collision avoidance). As a consequence, in this paper, we focus on the model identification for the DQSD by sufficiently considering perspective aspects of human, ship and circumstance.

3.5 Remark 2. From the entire model of DQSD and three submodels given by Equations (6), (14), (15), (21) and (24), respectively, we can straightforwardly find that the proposed DQSD model is essentially time-varying and therefore dynamic as the input variables of environment $\boldsymbol{\Omega}(t)$, ship manoeuvrability $\boldsymbol{\Theta}(t)$ and human state $\boldsymbol{\Xi}(t)$ 
Table 1. The principal dimensions of Esso Osaka tanker.

\begin{tabular}{lcc}
\hline Principal Dimensions & Parameters & Values \\
\hline Ship Length & $L(\mathrm{~m})$ & $304 \cdot 8$ \\
Ship Width & $B(\mathrm{~m})$ & $47 \cdot 17$ \\
Draft & $d(\mathrm{~m})$ & $18 \cdot 46$ \\
Displacement & $\nabla(\mathrm{m} 3)$ & 220000 \\
Block Coefficient & $C_{b}$ & $0 \cdot 83$ \\
Design Speed & $u_{0}(\mathrm{knot})$ & 16 \\
Nominal Revolution & $n_{0}(\mathrm{rpm})$ & 80 \\
\hline
\end{tabular}

Table 2. The principal dimensions of Esso Osaka tanker.

\begin{tabular}{|c|c|c|c|c|c|}
\hline \multicolumn{2}{|c|}{$\begin{array}{l}\text { Human } \\
\Xi=\left[\xi_{1}, \xi_{2}, \xi_{3}\right]^{\mathrm{T}}\end{array}$} & \multicolumn{2}{|c|}{$\underset{\Theta=\left[\boldsymbol{\Theta}_{S t a}, \boldsymbol{\Theta}_{D y n}\right] \mathrm{T}}{\text { Ship }}$} & \multicolumn{2}{|c|}{$\begin{array}{c}\text { Environment } \\
\mathbf{\Omega}=\left[\omega_{1}, \omega_{2}, \omega_{3}, \omega_{4}\right]^{\mathrm{T}}\end{array}$} \\
\hline Skill $\xi_{1}$ & {$[-1,0]$} & Stationary $\boldsymbol{\Theta}_{S t a}$ & {$\left[L, B, d, \nabla, C_{b}\right]$} & Visibility $\omega_{1}$ & {$[0,1]$} \\
\hline Physical $\xi_{2}$ & {$[-1,0]$} & Dynamic & $V \in[5 \mathrm{kn}, 25 \mathrm{kn}]$ & Wind $\omega_{2}$ & {$[0,1]$} \\
\hline \multirow[t]{2}{*}{ Mental $\xi_{3}$} & {$[-1,0]$} & $\boldsymbol{\Theta}_{D y n}=\left[V \delta_{0}\right]$ & $\delta_{0} \in\left[-35^{\circ}, 35^{\circ}\right]$ & Wave $\omega_{3}$ & {$[0,1]$} \\
\hline & & & & Congestion $\omega_{4}$ & {$[0,1]$} \\
\hline
\end{tabular}

are actually time-dependent functions. It follows that the DQSD model is a really dynamic framework for QSD-type ship domains.

3.6. Remark 3. Note that the proposed analytical framework for DQSD-type ship domains consists of three significant factors, i.e., human, ship and circumstance, whereby target ships in encounter situations would be exclusively investigated in other specific works concerning with DQSD-based collision avoidance and risk assessment. This coincides with the main idea in this paper that the DQSD framework is to achieve a general and fundamental model. In this case, without loss of generality, the open water area is considered as default situations to realise the backbone of the DQSD model. Actually, corresponding DQSD-type ship domains for restricted water areas (i.e., narrow channels, traffic separation zones and fairways, etc.) can be steadily obtained by incorporating the characteristics of restricted water areas into the general DQSD model.

4. SIMULATION STUDIES. In order to demonstrate the effectiveness of the proposed DQSD model, comprehensive simulation studies will be conducted on the well-known Esso Osaka tanker in several scenarios. The principal dimensions of Esso Osaka tanker are listed in Table 1. And, ship dynamic states are mainly considered as ship speed $V$ and command rudder angle $\delta_{0}$, where $V \in[5 \mathrm{kn}, 25 \mathrm{kn}]$ and $\left|\delta_{0}\right| \in\left[5^{\circ}, 35^{\circ}\right]$. Navigator state variables are selected as skill ability $\xi_{1}$, physical level $\xi_{2}$ and mental level $\xi_{3}$, where $\xi_{i} \in[-1,0]$. The environment states are chosen as visibility $\omega_{1}$, wind force $\omega_{2}$, wave $\omega_{3}$ and traffic congestion $\omega_{4}$, where $\omega_{i} \in[0,1]$. To be specific, the chosen parameters of human, ship and environment subsystems are shown in Table 2. Without loss of generality, we simply choose parameter $\boldsymbol{\Gamma}$ as $\boldsymbol{\Gamma}=[0 \cdot 25,0 \cdot 25$, $0 \cdot 25,0 \cdot 25]^{\mathrm{T}}$. 


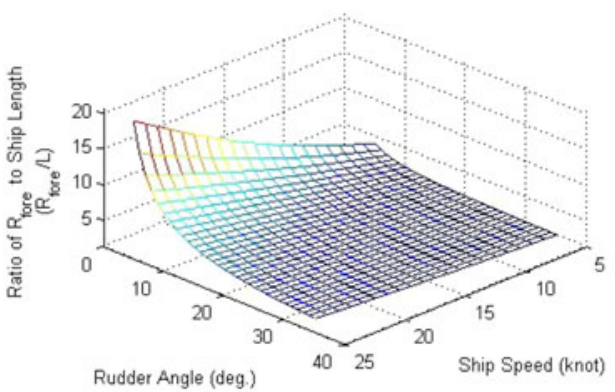

(a)

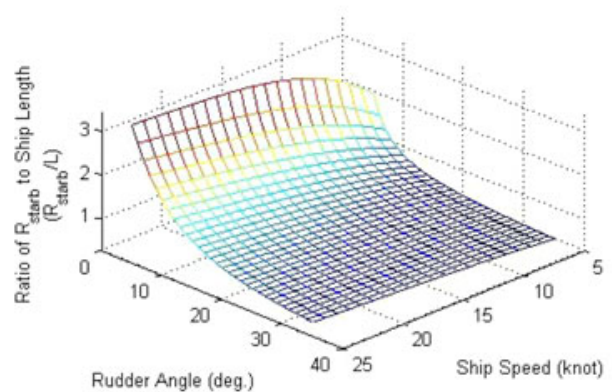

(c)

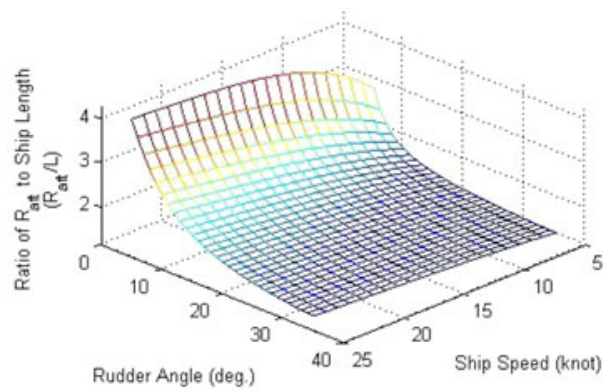

(b)

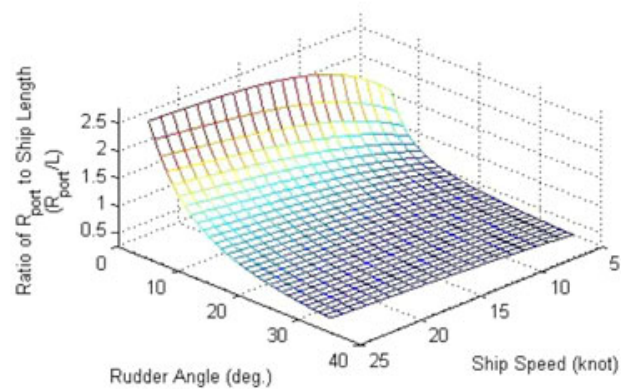

(d)

Figure 1. Simulation results for ship submodel of DQSD with ship speed and rudder angle being inputs, and (a) $R_{\text {fore }}$, (b) $R_{a f t}$, (c) $R_{\text {starb }}$ and (d) $R_{\text {port }}$ as outputs.

As the following simulation studies, the submodels of ship, human and environment will be investigated to validate the effectiveness of all the submodels which contribute to the integrated DQSD model. As a whole, typical situations will be studied to demonstrate the final performance of the DQSD model.

Firstly, simulation studies focus on the ship submodel of the DQSD model, whereby simulation results are shown in Figure 1. We can find that the scale parameters (domain radii) sharply decrease with increasing command rudder angle and decreasing ship speed. To be specific, the monotonous relationship becomes flat while the rudder angle exceeds $20^{\circ}$ and the ship speed falls below 15 knots, especially for the fore radius $R_{f o r e}$. The foregoing results apparently coincide with the subjective and objective practices in marine traffic.

Secondly, we consider the human submodel of DQSD model which identifies the ship navigator's factors including skill, physical and mental levels. Note that it is impossible to show the complete input-output relationship of the human submodel with three inputs and one output in three-dimensional space. Alternatively, we turn to visually present the underlying nonlinearity of the output $k$ partially dependent on each pair of input variables. The simulation results are shown in Figure 2, from which we can see that the shape parameter $k$ decreases with increasing inputs, skill level, physical and mental states, monotonously. It means that the shape of the DQSD would become 'fatter' and consequently conservative while the states of the navigator get worse. On the contrary, the shape would be much 'thinner' and progressive while the navigator's states become better. In addition, it should be noted that three inputs, i.e., skill level, physical and mental states, are considered to possess equal influences on 


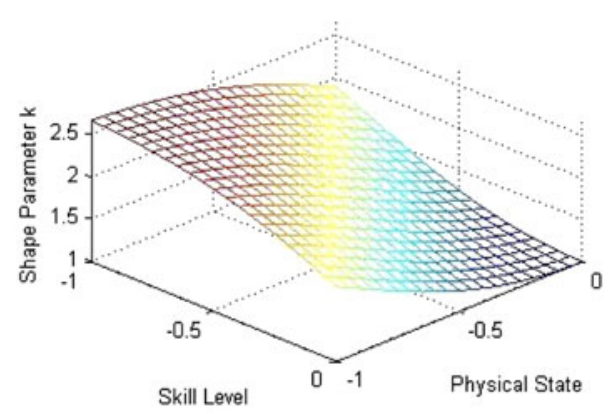

(a)

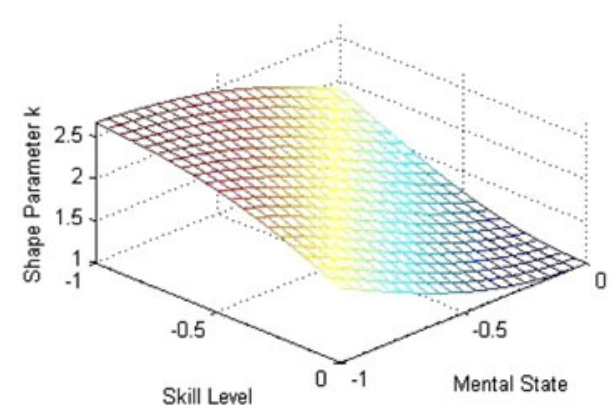

(b)

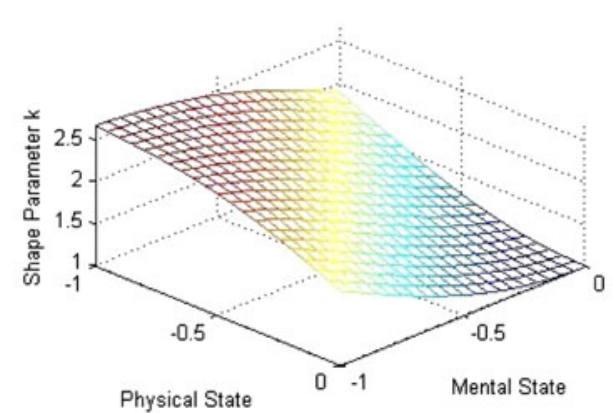

(c)

Figure 2. Simulation results for human submodel of DQSD with skill, physical and mental levels being inputs, and shape parameter $k$ as the output.

the shape of the DQSD. It obviously follows that the human submodel reveals the underlying navigator's characteristics affecting the ship domain model.

The circumstance submodel of the DQSD model is demonstrated to be effective via simulation studies, whereby the results are shown in Figure 3. Similar to the presentation methodology used in human submodel simulations, six pairwise visual presentations are also decomposed from the complete circumstance submodel with four input variables, i.e., visibility, wind force, wave and traffic congestion. Considering pairs of inputs for the circumstance submodel, the environment parameter $C$ partially dependent on two out of four input variables in the whole DQSD model (6) could be visualized in a straightforward manner. From Figure 3, it can be seen that the parameter $C$ is in inverse proportion to the environment state. To be specific, it reveals that the environment influence considered in this paper would be higher while the visibility becomes worse, and wind force, wave and traffic congestion become much more severe. As a result, the ship domain would be enlarged by increasing the parameter $C$ which is subject to the circumstance severity.

Furthermore, we conduct simulation studies on the whole DQSD model integrated by the foregoing ship, human and circumstance submodels. Without loss of generality, in order to clearly demonstrate the resulting DQSD model, we firstly consider several typical stationary situations that human factor parameters are selected as $\boldsymbol{\Xi}=[0,0,0]^{\mathrm{T}}$, $\boldsymbol{\Xi}=[-0 \cdot 2,-0 \cdot 2,-0 \cdot 2]^{\mathrm{T}}$ and $\boldsymbol{\Xi}=[-1,-1,-1]^{\mathrm{T}}$, ship speeds are selected as 15,20 and 25 knots, and circumstance parameters are selected as $\boldsymbol{\Omega}=[1,0,0,0]^{\mathrm{T}}$ and $\boldsymbol{\Omega}=[0,1,1,1]^{\mathrm{T}}$, respectively. The results are shown in Figure 4, from which we could find that the ship 

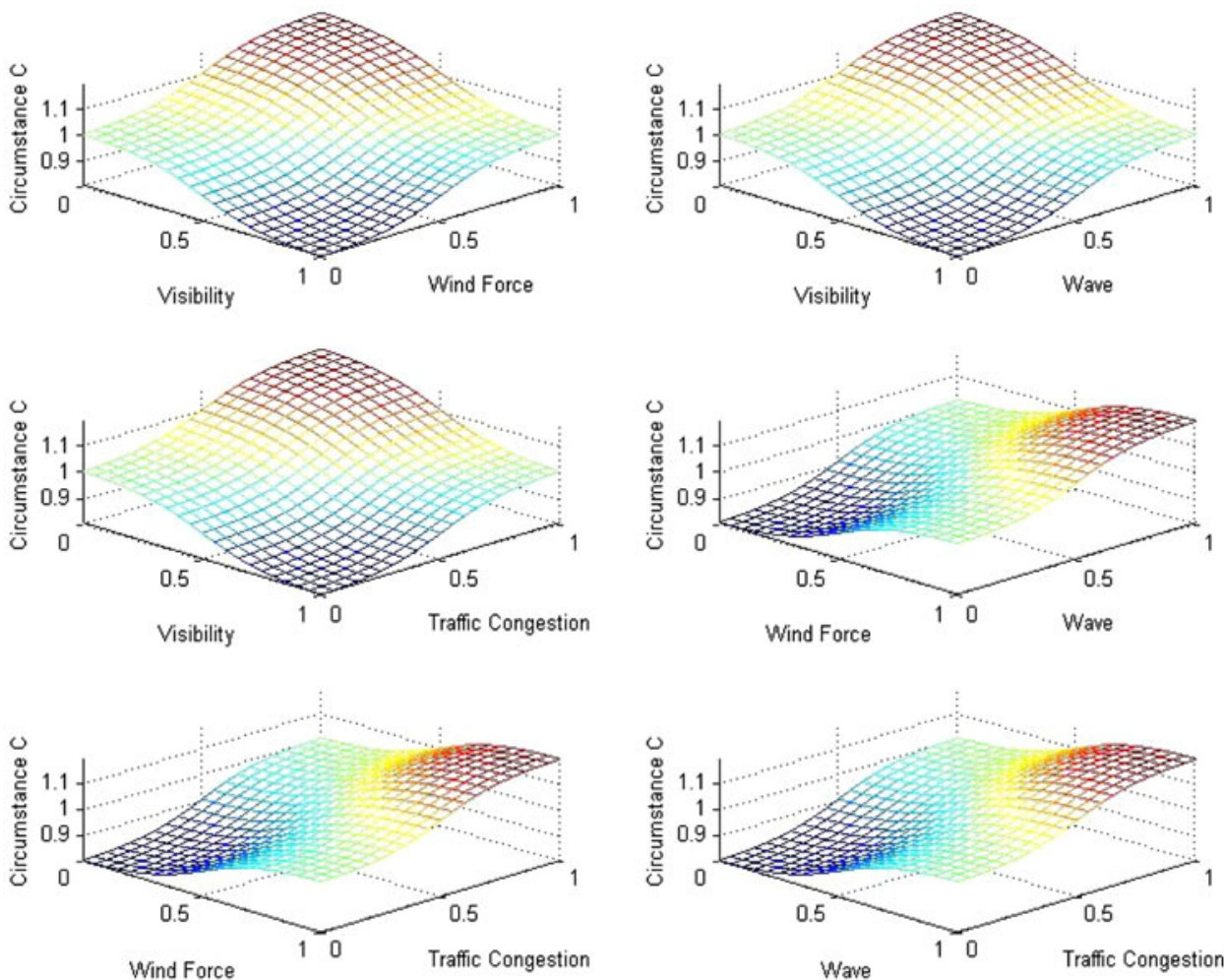

Figure 3. Simulation results for circumstance submodel of DQSD with visibility, wind force, wave and traffic congestion being inputs, and environment assessment $C$ as the output.

domain scale is determined by ship factors and circumstance states, respectively, while the ship domain shape is influenced by navigator's states. To be specific, on the one hand, the scale of the DQSD model would be increased with increasing ship speed and deteriorating circumstance states. On the other hand, the shape of the DQSD model would be increased with decreasing the navigator's abilities and levels. It follows that the abovementioned investigations are evidently identical to the essential characteristics of ship domains.

In order to demonstrate the dynamic performance of the entire DQSD model, we hereby consider arbitrary time-varying navigational situations whereby input signals are governed as follows: human factors $\Xi(t)=[-0 \cdot 5 \sin (0 \cdot 1 \pi t)-0 \cdot 5,0 \cdot 5 \sin (0 \cdot 5 \pi t)-0 \cdot 5$, $-0 \cdot 5 \sin (0 \cdot 4 \pi t)-0 \cdot 5]^{\mathrm{T}}$, ship speed $V(t)=15+10 \sin (0 \cdot 2 \pi t)$, command rudder angle $\delta_{0}(t)=35 \sin (0 \cdot 1 \pi t)$, and circumstance influences $\boldsymbol{\Omega}(t)=[0 \cdot 5 \sin (0 \cdot 5 \pi t)+0 \cdot 5,-0 \cdot 5 \sin$ $(0 \cdot 4 \pi t)+0 \cdot 5,0 \cdot 5 \sin (0 \cdot 3 \pi t)+0 \cdot 5,-0 \cdot 5 \sin (0 \cdot 2 \pi t)+0 \cdot 5]^{\mathrm{T}}$, respectively. Accordingly, simulation results of dynamic scale and shape parameters, i.e., $\mathbf{Q}(t)$ and $k(t)$, in the DQSD model are shown in Figure 5, from which we can see that the scale quaternion $\mathbf{Q}$ and shape index $k$ are dynamic simultaneously while time-varying perspective factors from human, ship and circumstance are considered. Specifically, the dynamics of the fore radius $R_{\text {fore }}$ tends to be significant in the scale quaternion of the DQSD model, and thereby identical to the reality that the fore sector of the ship domain is preferably kept clear. For the shape parameter $k$, the dynamic characteristics illustrate 


$$
\square=\left[\begin{array}{lll}
0 & 0 & \top
\end{array}\right]^{\top}-\Xi=\left[\begin{array}{lll}
-0.2-0.2-0.2 & ]^{\top}-\Xi=[-1-1 & -1
\end{array}\right]^{\top}
$$
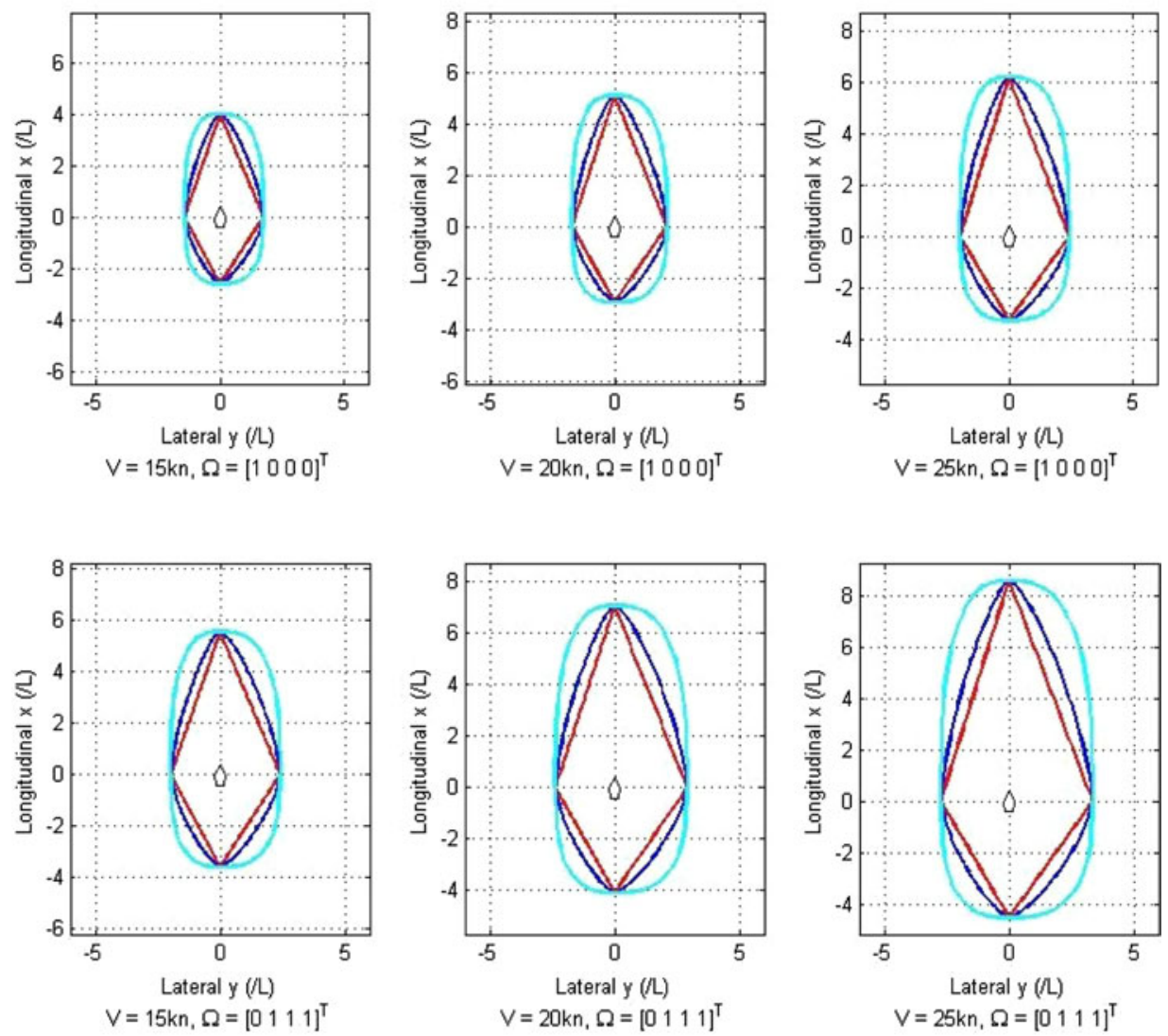

Figure 4. Simulation results for the DQSD with different stationary situations.

the conservatism of the current DQSD model which is mainly related to the navigator states. The resulting dynamic performances of the DQSD model are shown in Figure 6, whereby ten discrete DQSD-type ship domains are depicted along the time axis. It can be seen that the DQSD model can be able to dynamically reflect timevarying situations. Actually, the continuous dynamics of the DQSD model could be considered as a set of instant stationary DQSD-type ship domains corresponding to each time point. As shown in Figure 4, the effectiveness of DQSD models with stationary situations has been clearly evaluated. In addition, both scales and shapes of the DQSD model are adaptive to changing input signals from human, ship and circumstance, respectively. It follows that the DQSD-type ship domains achieve convincing dynamic performances.

5. CONCLUSIONS. In this paper, a novel Dynamic Quaternion Ship Domain (DQSD) has been proposed to establish an analytical framework for reasonable ship domain models possessing objective and subjective characteristics. Unlike previous 


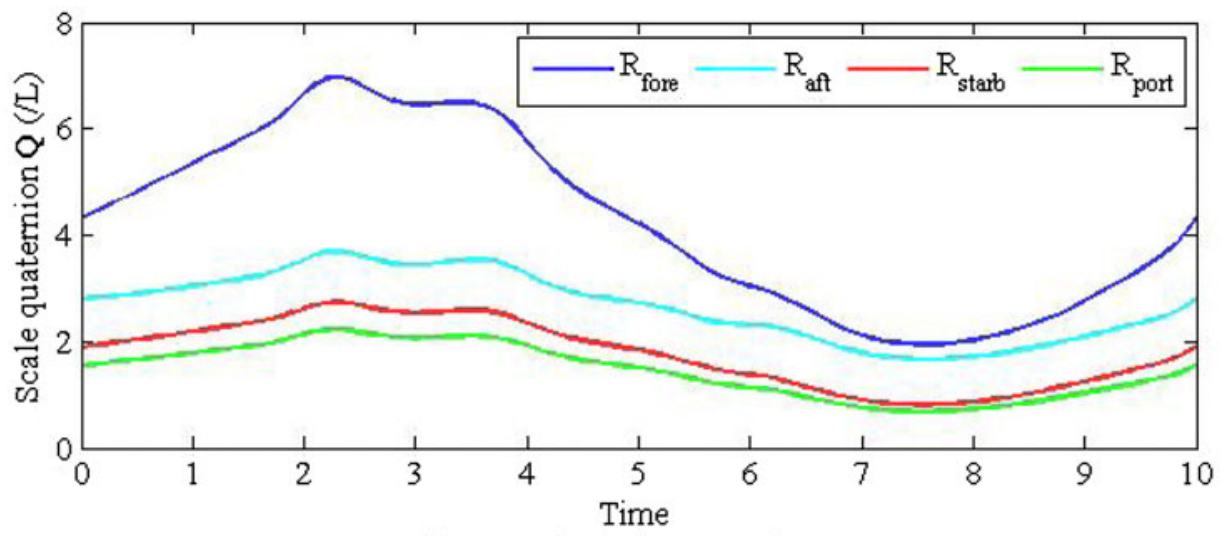

(a) Dynamic scale quaternion $Q$

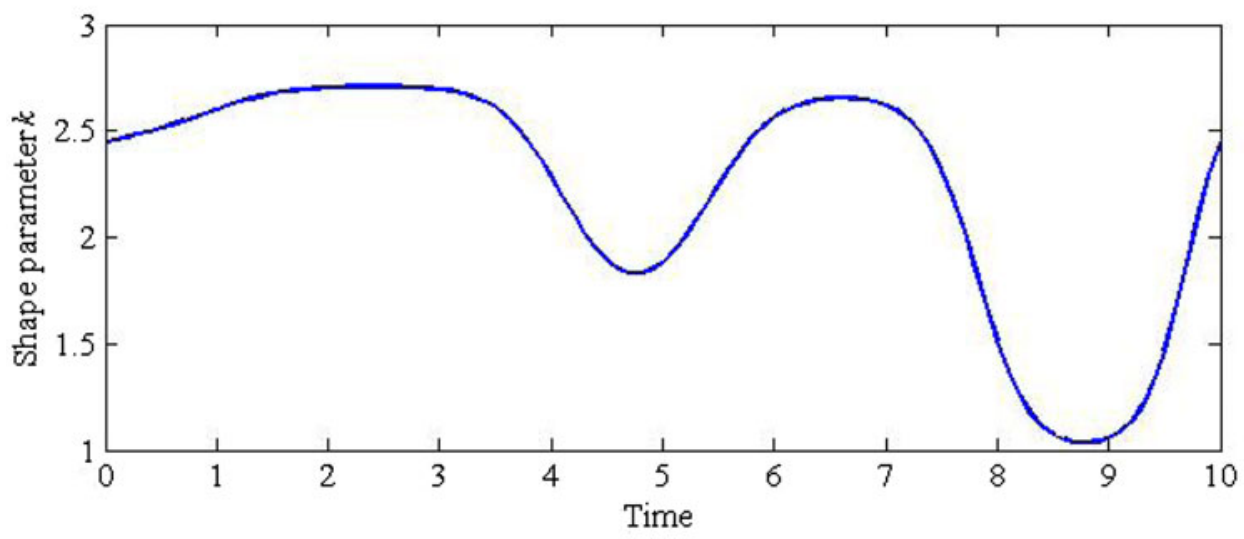

(b) Dynamic shape parameter $k$

Figure 5. The dynamic scale and shape parameters of the DQSD model.

geometry ship domains (i.e., circle, ellipse and polygon ship domains), the DQSD is modelled in an analytical manner on the basis of the Quaternion Ship Domain (QSD) model. To be specific, the proposed DQSD model is parameterized by: the scale vector Q; the shape parameter $k$ and the zooming coefficient $C$, where the longitudinal and lateral dimensions of the DQSD are determined by vector $\mathbf{Q}$ subject to ship manoeuvrability; the shape of the DQSD is defined by parameter $k$ derived from navigator's states; and the coefficient $C$ obtained by navigation circumstance is used to zoom in or out the corresponding DQSD-type ship domain. It should be noted that the human, ship and circumstance submodels of the DQSD are well defined by sufficiently taking into account pervasive influence factors, respectively. Furthermore, the entire DQSD model integrated by above mentioned submodels has been well implemented in an analytical framework for ship domains. As a consequence, the proposed DQSD model can effectively identify objective and subjective information from the aspects of human, ship and circumstance. It follows that the resulting DQSD model is adequate candidate for complex ship domain identifications. In order to demonstrate the effectiveness of the proposed DQSD method, simulation studies have 


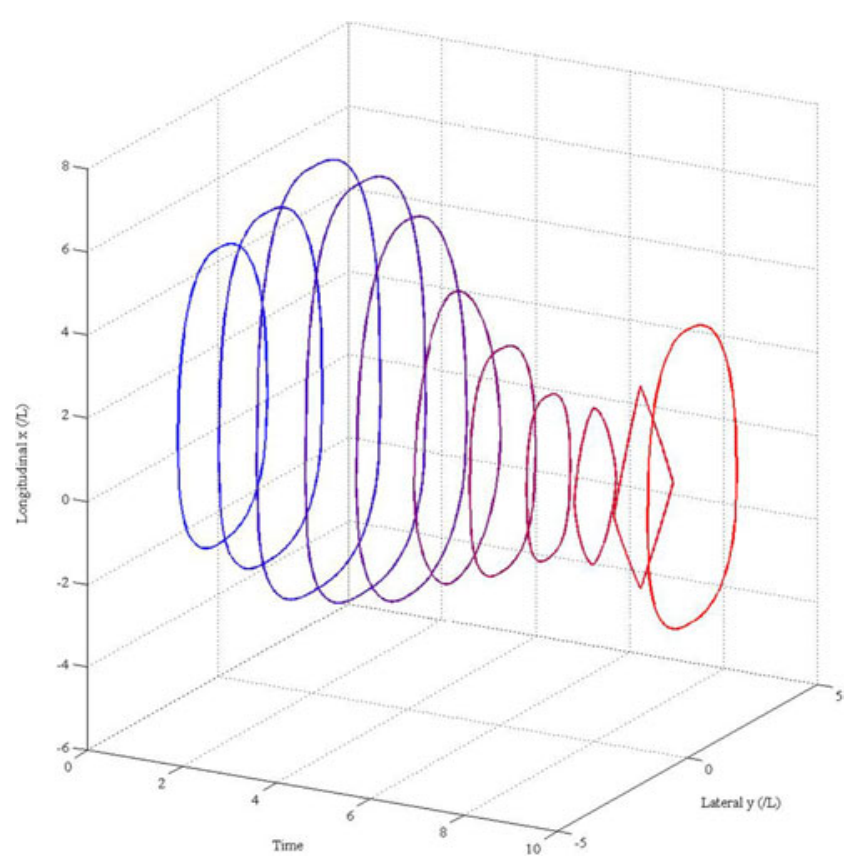

Figure 6. Dynamic simulation results for the DQSD model with time-varying situations.

been conducted on the well-known Esso Osaka tanker in typical stationary and dynamic situations. The results indicate that the human, ship and circumstance submodels coincide with the practical facts, respectively, and the integrated DQSD model is able to capture the essential characteristics of ship domains and thereby effective and superior to previous ship domains in terms of performance and accuracy. It should be highlighted that the proposed analytical framework for DQSD models achieve dynamic performances adaptive to time-varying situations, and therefore provide marine traffic engineering, collision avoidance, path planning and ship motion control, etc., with fundamental principles and effective tools, which would be presented in future work.

\section{ACKNOWLEDGEMENTS}

This paper is financially supported by National Nature Science Foundation of China (under Grant 51009017), Applied Basic Research Funds from Ministry of Transport of the People's Republic of China (2012-329-225-060), and Fundamental Research Funds for the Central Universities of China (under Grant 2009QN025 and 2011JC002).

\section{REFERENCES}

Coldwell, T. G. (1983). Marine Traffic Behaviour in Restricted Waters. The Journal of Navigation, 36, 431-444.

Davis, P. V., Dove, M. J. and Stockel, C. T. (1980). A Computer Simulation of Marine Traffic Using Domains and Arenas. The Journal of Navigation, 33, 215-222.

Davis, P. V., Dove, M. J. and Stockel, C. T. (1982). A Computer Simulation of Multi-Ship Encounters. The Journal of Navigation, 35, 347-352. 
Fujii, Y. and Tanaka, K. (1971). Traffic Capacity. The Journal of Navigation, 24, 543-552.

Goodwin, E. M. (1975). A Statistical Study of Ship Domains. The Journal of Navigation, 28, 329-341.

Gucma, L. and Pietrzykowski, Z. (2006). Ship Manoeuvring in Restricted Areas: an Attempt to Quantify Dangerous Situations Using a Probabilistic-Fuzzy Method. The Journal of Navigation, 59, 251-262.

Hwang, C. N. (2002). The Integrated Design of Fuzzy Collision-Avoidance and $H_{\infty}$-autopilots on Ships. The Journal of Navigation, 55, 117-136.

Kao, S. L., Lee, K. T., Chang, K. Y. and Ko, M. D. (2007). A Fuzzy Logic Method for Collision Avoidance in Vessel Traffic Service. The Journal of Navigation, 60, 17-31.

Kijima, K. and Furukawa, Y. (2001). Design of Automatic Collision Avoidance System Using Fuzzy Inference. Proceeding of IFAC Conference on Control Applications in Marine Systems, Glasgow, U.K.

Kijima, K. and Furukawa, Y. (2003). Automatic Collision Avoidance System Using the Concept Of Blocking Area. Proceeding of IFAC Conference on Manoeuvring and Control of Marine Craft, Girona, Spain.

Lisowski, J., Rak, A. and Czechowicz, W. (2000). Neural Network Classifier for ship domain assessment. Mathematics and Computers in Simulation, 51, 399-406.

Liu, S. M., Wang, N. and Wu, Z. L. (2011). Review of Research on Ship Domain. Journal of Dalian Maritime University, 37(1), 51-54. (in Chinese)

Pedersen, E. and Inoue, K. (2003). Simulator Studies on a Collision Avoidance Display that Facilitates Efficient and Precise Assessment of Evasive Manoeuvres in Congested Waterways. The Journal of Navigation, 56, 411-427.

Pietrzykowski, Z. and Uriasz, J. (2004). The Ship Domain in a Deep-Sea Area. Proceeding of the $3^{\text {rd }}$ International Conference on Computer and IT Applications in the Maritime Industries, Siguenza, Spain.

Pietrzykowski, Z. and Uriasz, J. (2006). Ship Domain in Navigational Situation Assessment in an Open Sea Area. Proceeding of the $5^{\text {th }}$ International Conference on Computer and IT Applications in the Maritime Industries, Oegstgeest, Netherlands.

Pietrzykowski, Z. and Uriasz, J. (2009). The Ship Domain - A Criterion of Navigational Safety Assessment in an Open Sea Area. The Journal of Navigation, 62, 93-108.

Pietrzykowski, Z. (2008). Ship's Fuzzy Domain - A Criterion For Navigational Safety in Narrow Fairways. The Journal of Navigation, 61, 499-514.

Qu, X., Meng, Q. and Li, S. (2011). Ship Collision Risk Assessment for the Singapore Strait. Accident Analysis \& Prevention, 43(6), 2030-2036.

Smierzchalski, R. and Michalewicz, Z. (2000). Modelling of a Ship Trajectory in Collision Situations at Sea by Evolutionary Algorithm. IEEE Transaction on Evolutionary Computation, 4(3), 227-241.

Smierzchalski, R. (2001). On-line Trajectory Planning in Collision Situation at Sea by Evolutionary Computation-Experiments. Proceeding of IF AC Conference on Computer Applications in Marine Systems, Glasgow, U.K.

Szlapczynski, R. (2006). A Unified Measure of Collision Risk Derived From the Concept of a Ship Domain. The Journal of Navigation, 59, 477-490.

Szlapczynski, R. (2008). A New Method of Planning Collision Avoidance Manoeuvres for Multi-Target Encounter Situations. The Journal of Navigation, 61, 307-321.

Wang, N., Meng, X. Y., Xu, Q. Y. and Wang, Z. W. (2009). A Unified Analytical Framework for Ship Domains. The Journal of Navigation, 62, 307-321.

Wang, N. (2010). An Intelligent Spatial Collision Risk Based on the Quaternion Ship Domain. The Journal of Navigation, 63, 733-749.

Wang, N., Tan, Y. and Liu, S. M. (2011) Ship Domain Identification Using Fast and Accurate Online Self-organizing Parsimonious Fuzzy Neural Networks. Proceedings of Chinese Control Conference, Yantai, China.

Wilson, P. A., Harris, C. J. and Hong, X. (2003). A Line of Sight Counteraction Navigation Algorithm for Ship Encounter Collision Avoidance. The Journal of Navigation, 56, 111-121.

Zhao, J., Wu, Z. and Wang, F. (1993). Comments on ship domains. The Journal of Navigation, 46, 422-436.

Zhu, X., Xu, H. and Lin, J. (2001). Domain and its Model Based on Neural Networks. The Journal of Navigation, 54, 97-103. 